\title{
Zonal Flow Regime Changes in a GCM and in a Simple Quasigeostrophic Model: The Role of Stratospheric Dynamics
}

\author{
ISABELLA BORDI \\ Department of Physics, Sapienza University of Rome, Rome, Italy \\ KLAUS FRAEDRICH \\ Meteorologisches Institut, Universität Hamburg, and Max-Planck-Institut für Meteorologie, Hamburg, Germany \\ MICHAEL GHIL* \\ Geosciences Department, and Laboratoire de Météorologie Dynamique (CNRS and IPSL), Ecole Normale Supérieure, Paris, France \\ Alfonso SUTERA \\ Department of Physics, Sapienza University of Rome, Rome, Italy \\ (Manuscript received 22 February 2008, in final form 16 October 2008)

\begin{abstract}
The atmospheric general circulation is characterized by both single- and double-jet patterns. The doublejet structure of the zonal mean zonal wind is analyzed in Southern Hemisphere observations for the two calendar months of November and April. The observed features are studied further in an idealized quasigeostrophic and a simplified general circulation model (GCM). Results suggest that capturing the bimodality of the zonal mean flow requires the parameterization of momentum and heat fluxes associated with baroclinic instability of the three-dimensional fields.

The role of eddy heat fluxes in generating the observed double-jet pattern is ascertained by using an analytical Eady model with stratospheric easterlies, in which a single wave disturbance interacts with the mean flow. In this model, the dual jets are generated by the zonal mean flow correction. Sensitivity of the results to the tropospheric vertical wind shear (or, equivalently, the meridional temperature gradient in the basic state's troposphere) is also studied in the Eady model and compared to related experiments using the simplified GCM.
\end{abstract}

\section{Introduction}

The variability of the general circulation in the Southern Hemisphere ( $\mathrm{SH}$ ) has been studied fairly intensively over the last two decades as satellites have provided more abundant upper-air data (Mo and Ghil 1987). Many studies confirm the intuition that the much weaker

\footnotetext{
* Additional affiliation: Department of Atmospheric and Oceanic Sciences, and Institute of Geophysics and Planetary Physics, University of California, Los Angeles, Los Angeles, California.

Corresponding author address: Alfonso Sutera, Department of Physics, Sapienza University of Rome, Piazzale Aldo Moro 2, 00185 Rome, Italy.

E-mail: alfonso.sutera@roma1.infn.it
}

topographic forcing and land-sea contrast in this hemisphere lead to a circulation that-unlike in the Northern Hemisphere $(\mathrm{NH})$-is prevalently zonal (Kidson 1988). Consequently, these studies are mainly focused on the analysis of the low-frequency variability of zonal mean flow anomalies.

Yoden et al. (1987) analyzed four years (1980-83) of National Meteorological Center (NMC) data and identified two distinct regimes that correspond to single- and double-jet states, each of which persisted with a characteristic duration of a month. More recently, Hartmann and Lo (1998), using 10 years of European Centre for Medium-Range Weather Forecasts (ECMWF) analyses, showed that the eddies act to sustain the zonal flow anomalies and can lead to a jet pattern that alternates between a single broad jet and dual maxima in the 
upper troposphere. Koo et al. (2002), using the National Centers for Environmental Prediction (NCEP)-National Center for Atmospheric Research (NCAR) reanalysis dataset (Kalnay et al. 1996), found that these anomalies have considerable low-frequency variability, with peaks near 135 and 70 days. This variability manifests itself as an almost intransitive switching between two regimes, characterized by a displacement of the main jet axis with respect to its climatological position.

Koo et al. (2002) found that the two regimes are maintained by transient eddy forcing against surface friction. Attempts to explain variations in zonal flow intensity and zonal jet shifts in the NH (Rossby et al. 1939; Namias 1950; Kravtsov et al. 2006), as well as in the SH (see references above), have used two- or threelayer quasigeostrophic channel models [for an $f$-plane geometry, see Lorenz (1963); for $\beta$-plane studies, see Koo and Ghil (2002); Kravtsov et al. (2005), and references therein] and primitive equation models with various degrees of vertical resolution (e.g., Yu and Hartmann 1993; Akahori and Yoden 1997).

In the present paper, we focus on the SH zonal mean circulation for two particular months, November and April, when the climatological mean meridional circulation is almost symmetric with respect to the equator. This feature allows us to compare observations with the simulations of a simplified general circulation model (GCM) in an equatorially symmetric configuration. Moreover, a cursory inspection of the climatology of the zonal-mean zonal wind for each month of the year (see Fig. 1 of Hartmann and Lo 1998) shows a clear tropospheric double-jet structure in these two months (see also Gallego et al. 2005); the origin of this structure is the subject of our investigation. Multiple-jet patterns have been also observed on the major planets, as well as in the rotating, differentially heated annulus (see the review paper by Galperin et al. 2006, and references therein). These additional findings reinforce our interest in how this monthly mean feature is sustained in Earth's $\mathrm{SH}$.

A multiple-jet structure may be obtained in a forced barotropic flow (Farrell and Ioannou 2003) or in a weakly unstable baroclinic flow driven by thermal relaxation (Charney 1973). A common feature of both flows is a finite meridional scale of the eddy field. On a sphere, or even in a channel with large width, the meridional scale of the eddy field is finite, so that multiple jets are expected to be the prevalent solution, in particular for a basic-flow pattern that leads to weakly unstable waves (see, e.g., Panetta 1993 and Williams 2003).

In the case of an Eady problem, Bordi et al. (2002) have shown that the marginal instability curve is con- siderably modified for a baroclinic flow in which the zonally symmetric thermal forcing above the tropopause has a reversed equator-to-pole temperature gradient that gives rise to stratospheric easterlies. In this case, the Eady growth rate, all else being the same, decreases so that the system is closer to a state of weak instability. Bordi et al. (2006) have focused on the key role of the stratosphere in the baroclinic adjustment in a nonlinear Eady problem. They studied the wave-mean flow interaction by considering a single mode in the eddy field and in the zonal mean correction and showed that the stratospheric flow introduces a considerable amount of variability in the model dynamics. Bordi et al. (2007) found that this link between the stratosphere and troposphere modulated the variability of the tropospheric double-jet structure observed in the Northern Hemisphere winter. By considering this theoretical framework, together with the abovementioned observations, we propose that a reversal of the meridional temperature gradient in the $\mathrm{SH}$ stratosphere may affect the eddy field that generates and maintains the doublejet regime.

The objective of the present paper is therefore to revisit the effect of the baroclinic eddies and, more specifically, to study the role played by stratospheric easterlies in giving rise to observed salient features of the SH's zonally averaged monthly-mean circulation, such as the double-jet structure. For this purpose we analyze solutions of a simplified GCM forced by Newtonian cooling. The sensitivity of the zonal mean circulation to changes in the meridional temperature gradient in the troposphere, imposed by the external radiative constraint, is studied. Moreover, numerical solutions of a highly truncated nonlinear Eady model with a two-layer representation of the troposphere and stratosphere are also analyzed. To capture the doublejet pattern, we modify the model setup of Bordi et al. (2006) and consider a single mode in the wave field but two meridional modes in the zonal mean correction. The main purpose of the investigations based on the nonlinear Eady model is to isolate and highlight the role of the heat transports in generating a double-jet zonal wind structure; comparisons between the Eady solutions, the GCM solutions and observations are less crucial, given the extreme simplicity of the Eady model.

The paper is organized as follows: In section 2 the observed zonal mean circulation for November and April is presented. In section 3 we analyze the numerical solutions of our simplified GCM. Different thermal structures of the external radiative forcing in the stratosphere are applied to the model atmosphere and its full solutions are compared to the axisymmetric ones. Furthermore, the sensitivity of the model solutions to 
the forcing induced by the tropospheric equator-to-pole temperature difference is studied. This sensitivity study may be interpreted in the context of both climate variability and climate change because radiative forcing varies on intraseasonal as well as on interdecadal time scales (Kushner et al. 2001; Stone et al. 2001). In section 4 , we analyze the role of eddy heat fluxes in giving rise to the double-jet pattern by using a nonlinear Eady model with a highly idealized stratosphere. This analysis includes a sensitivity study of the zonal wind to the vertical wind shear in the troposphere. A summary and discussion are presented in section 5. A detailed description of the two-layer Eady model is provided in the appendix.

\section{Observations}

\section{a. Data}

The observed main features of the general circulation presented here are based on the 40-yr ECMWF ReAnalysis (ERA-40) data for September 1957-August 2002 (Uppala et al. 2005) available on a $2.5^{\circ} \times 2.5^{\circ}$ regular latitude-longitude grid, with 23 pressure levels ranging from $1000 \mathrm{hPa}$ up to $1 \mathrm{hPa}$. Comparing these observations with solutions of a simplified GCM, which are symmetric with respect to the equator, has led us to select the months of November and April for in-depth analysis because their climatological mass streamfunctions show the required symmetry to a good approximation. The Stokes mass streamfunction $\Psi$ is computed by downward trapezoidal integration of the meridional mass flux at all available vertical levels; we apply a small correction at the four lowest levels to ensure that $\Psi=0$ at the bottom boundary, as in Dima and Wallace (2003).

\section{b. Analysis}

In the midspring and midfall months of November and April (1958-2001), the SH monthly mean circulation is characterized by a main westerly jet near $50^{\circ} \mathrm{S}$, with a maximum intensity at $250 \mathrm{hPa}$, and a secondary jet around $30^{\circ} \mathrm{S}$, with its maximum at $200 \mathrm{hPa}$ (Figs. 1a,b). Note that easterlies dominate the tropical and midlatitude stratosphere, where they are particularly strong compared to near-surface easterlies in the tropics. The associated mass streamfunctions (Figs. 1c,d) reveal the typical three-cell structure of the earth's troposphere: an intense Hadley cell extending from the equator to about $35^{\circ} \mathrm{S}$, a Ferrell cell in the midlatitudes, and a weak polar cell at high latitudes. Furthermore, a weak connection between the thermally direct Hadley and polar cells extends throughout the lower stratosphere and is clearly visible for November, whereas there is just a hint of a poleward tilt of the Hadley cell at high levels in April. Bordi et al. (2007) first pointed out and commented on this feature and we will not discuss it here further.

On a daily basis, the upper tropospheric double-jet pattern is associated with synoptic variability: A latitudetime section of the zonal-mean zonal wind at $200 \mathrm{hPa}$ for April 2000 (Fig. 2b) displays two coexisting jets with intermittent amplification of the secondary one near $30^{\circ} \mathrm{S}$, while the monthly mean field (Fig. 2a) is quite similar to the climatological field in Fig. 1b; similar features appear for this month in other years as well (not shown). This intermittent variability suggests that the physical mechanism leading to the double-jet pattern is baroclinic instability, with its associated eddyinduced heat and momentum fluxes. In the next section, simplified GCM experiments demonstrate that eddies modulated by stratospheric thermal gradient reversal can establish multiple tropospheric jets in agreement with the observations.

\section{Numerical results with a simplified GCM}

\section{a. Model and parameter values}

The Portable University Model of the Atmosphere (PUMA; available online at www.mi.uni-hamburg.de/ puma; Fraedrich et al. 1998, 2005) is a simplified GCM. Its dynamical core is a global spectral model solving the primitive equations on sigma levels (Hoskins and Simmons 1975; James and Gray 1986). The diabatic heating rate is represented through Newtonian cooling and the dissipative processes by Rayleigh friction, which may act on each model level (see also Held and Suarez 1994). The model was run at a T21 resolution with 20 equally spaced $\sigma$ levels, following the analysis by Bordi et al. (2007) on tropospheric double jets in Northern Hemisphere winter.

The 20 vertical levels are a compromise between the need for sufficient resolution in the stratosphere and maintaining a reasonable computational burden. After testing a model version with 40 vertical levels, we concluded that 20 levels in the vertical, with five above the tropopause, are sufficient for our purpose, which is merely to impose a potential vorticity gradient across the tropopause rather than a rigid lid. Possible reflection of vertically propagating waves at the upper boundary is well known to affect the details of stratospheretroposphere interactions (which are not a matter of concern for us here) but did not seem to affect the tropospheric jet dynamics of interest in the present paper.

The general atmospheric circulation is studied here in terms of model solutions for steady external radiative forcing. At each time step the model temperature is 

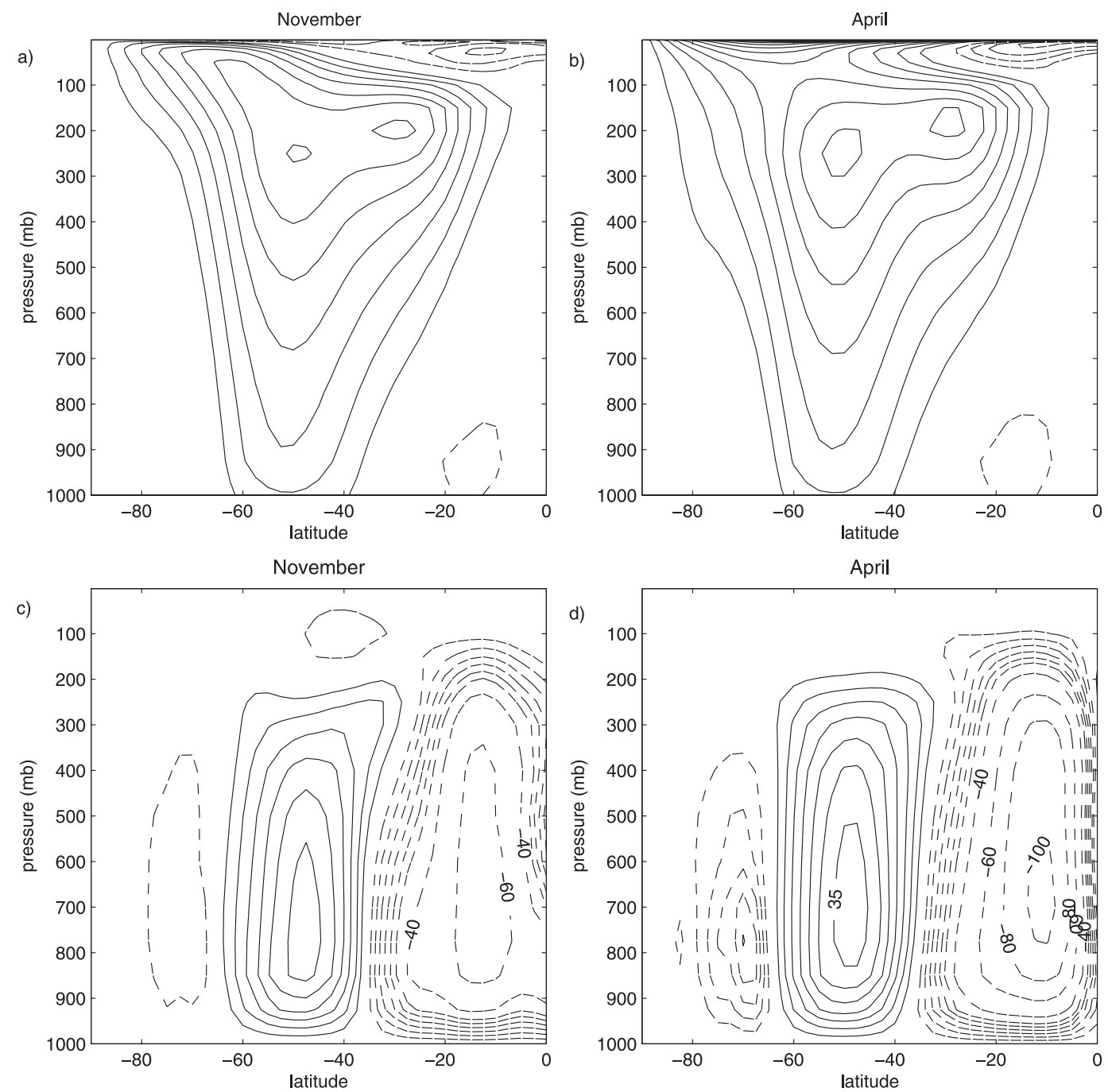

FIG. 1. Observed climatological field for the SH: (a),(b) latitude-height (pressure) cross section of the zonalmean zonal wind for November (austral spring) and April (austral fall), respectively; (c),(d) zonal-mean cross section of the mass streamfunction for November and April, respectively. Units in (a) and (b) are $\mathrm{m} \mathrm{s}^{-1}$, contours are every $4 \mathrm{~m} \mathrm{~s}^{-1}$, and the zero line is excluded. In (c) and (d), units are $10^{9} \mathrm{~kg} \mathrm{~s}^{-1}$, contours are every $5 \times 10^{9} \mathrm{~kg} \mathrm{~s}^{-1}$ within the range $(-30,30)$, and the zero line is excluded; values greater than $30 \times 10^{9} \mathrm{~kg} \mathrm{~s}^{-1}$ and less than $-40 \times 10^{9}$ $\mathrm{kg} \mathrm{s}^{-1}$ are shown with labels. Dashed lines in (a)-(d) denote negative values and solid lines positive ones.

relaxed toward a prescribed restoring temperature field $T_{r}$, describing hemispherically symmetric conditions, that is obtained by interpolating the idealized equinox forcing introduced by Akahori and Yoden (1997) to PUMA's $\sigma$ levels (Bordi et al. 2007). The radiative forcing as a function of latitude and sigma level is

$$
T_{r}(\phi, \sigma)=T_{0}(\sigma)+\frac{\Delta T_{r}(\sigma)}{2}\left(\cos 2 \phi-\frac{1}{3}\right)
$$

where $T_{0}(\sigma)$ is the global mean temperature at each level and $\Delta T_{r}(\sigma)$ is the equator-to-pole temperature difference. Note that $\Delta T_{r}(\sigma)<0$ implies a reversed meridional temperature gradient; in the present study, this gradient is applied to the model's four uppermost levels (see Table 1) to allow the jet to decrease with lower altitude in the stratosphere, as the observations suggest.

The relaxation time for the Newtonian forcing is 15 days for all levels, with the exception of the uppermost level, where it is set to 7.6 days. A hyperdiffusion of the form $K \nabla^{8}$, with $K$ being the diffusion coefficient, is applied to the temperature, divergence, and vorticity equations to prevent accumulation of enstrophy at the shortest scales and to preserve numerical stability. 

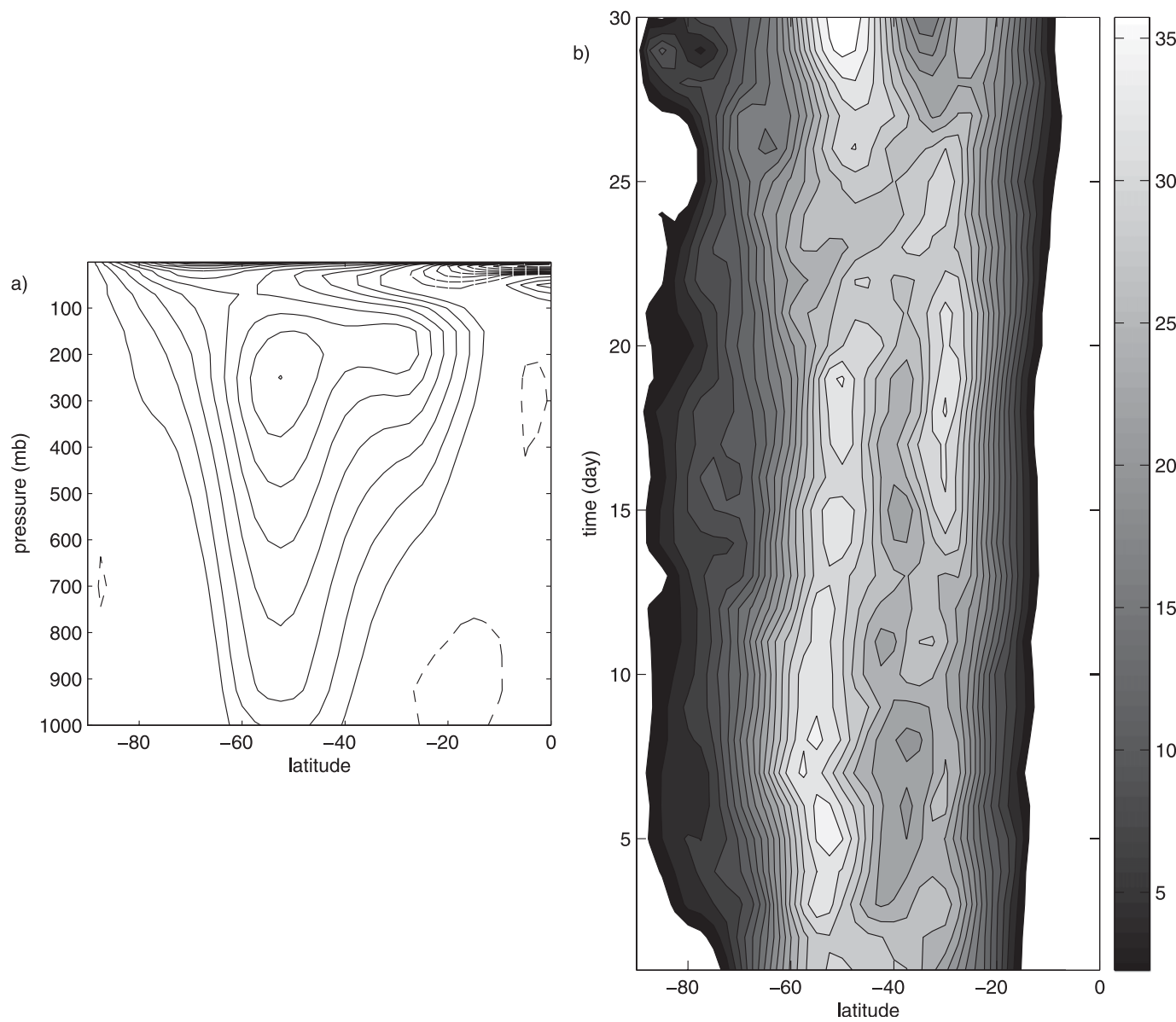

FIG. 2. Observed SH fields for April 2000: (a) latitude-height (pressure) cross section of the monthly mean zonal wind and (b) latitude-time diagram of the zonal mean zonal wind at $200 \mathrm{hPa}$. Units and contours in (a) are the same as in Figs. 1a,b.

Divergence and vorticity are damped at the lowermost level with a Rayleigh friction time scale $\tau_{R}$ of 0.1 days. The model can start from a restart file or from an atmosphere at rest; we use the second option, with the initial state a motionless, stably stratified atmosphere.

The model is formulated so that only modes symmetric with respect to the equator are allowed. We perform runs with and without eddies: in both cases the atmosphere starts from rest, but for the eddy-permitting runs, we introduce an initial random perturbation that is not zonally symmetric. Furthermore, when explicitly mentioned, a vertical diffusion with coefficient $\nu=1 \mathrm{~m}^{2} \mathrm{~s}^{-1}$ is applied. To isolate the role of baroclinic instability, we intentionally neglect any form of convection, land-sea contrast, or topography.

TABLE 1. Experimental parameter values for PUMA: the global mean restoring temperature $T_{0}(\sigma)$ and the equator-to-pole temperature difference $\Delta T_{r}(\sigma)$.

\begin{tabular}{ccc}
\hline \hline Experiment & $T_{0}(\sigma)(\mathrm{K})$, top to bottom & $\Delta T(\sigma)(\mathrm{K})$, top to bottom \\
\hline $\mathrm{C} 1$ & $265.14,254.91,246.36,240.95,237.56,234.65,235.24$, & $0.00,-25.05,-64.32,-58.19,-22.13,28.26$, \\
& $237.73,243.54,248.87,253.41,257.83,261.72$, & $34.91,40.37,45.10,49.19,52.05,54.69,56.20$, \\
$\mathrm{C} 2$ & $265.60,268.96,272.33,275.36,278.34,281.06,283.74$ & $57.70,58.31,58.91,59.26,59.56,59.76,60.00$ \\
& As for C1 & $0.00,0.00,0.00,0.00,0.00,28.26,34.91,40.37$, \\
$\mathrm{C} 3$ & As for C1 & $45.10,49.19,52.05,54.69,56.20,57.70,58.31,58.91$, \\
& & $59.26,59.56,59.76,60.00$ \\
\end{tabular}




\section{b. Experimental setup}

We designed three PUMA experiments to illustrate the effects of the eddies and the role of the stratosphere in generating the tropospheric double-jet pattern (see Table 1 for the exact parameter settings). Common to all experiments is the global mean temperature profile in the troposphere, with a tropopause height at $275 \mathrm{hPa}$.

The first experiment is labeled $\mathrm{C} 1$ and provides the no-eddy simulation. A reversed stratospheric meridional temperature gradient is set up and vertical diffusion is introduced to make the model simulation comparable with the Held and Hou (1980) analysis of nonlinear, axially symmetric circulations. The second experiment (C2) has the same meridional temperature gradient in the troposphere, whereas in the stratosphere it is set to zero. In this case we consider only the effect of the eddies and no vertical diffusion. In the third experiment (C3) we introduce a reversed meridional temperature gradient in the stratosphere as in $\mathrm{C} 1$, as well as allowing eddies to form, and thus study the combined effect of the eddies and of the reversed stratospheric gradient. In this case, as in experiment $\mathrm{C} 2$, vertical diffusion is neglected.

Prior to the analysis of these three experiments, longtime simulations with no eddies and no vertical diffusion were carried out (not shown). These preliminary experiments helped us establish the key effects of eddies and vertical diffusion in generating the zonal mean circulation: For a stratosphere with a null meridional temperature gradient, the model simulation converges to a superrotating atmosphere in thermal wind balance, with zero meridional velocity and westerlies at the equator. When the stratospheric temperature gradient is reversed, the simulation leads to a westerly jet centered at the equatorial tropopause, with an upwarddecreasing intensity.

For all three PUMA experiments in the present paper, the statistics of the flow stabilize after $2-3 \mathrm{yr}$; hence, the results are presented for the fourth year. The zonalmean zonal wind fields for the three cases are displayed in Fig. 3. Hereafter we denote by $\Delta T$ the imposed equator-to-pole temperature difference at the lowest model level; that is, $\Delta T=\Delta T_{r}(\sigma=1)$.

We discuss the results for $\Delta T=60 \mathrm{~K}$ (Figs. 3b,e,h) first. Experiment $\mathrm{C} 1$ leads to a steady-state solution characterized by a single jet, with its core near $25^{\circ} \mathrm{S}$ and near the tropopause. Wind speeds decrease away from this core, toward the pole and surface and, more rapidly, toward the equator and the stratosphere. This result conserves angular momentum, as in the Held and Hou (1980) theory of the axisymmetric circulation, albeit in the presence of a reversed stratospheric temperature gradient. The main shortcoming of this solution, though, is that no double jet is produced.

When eddies are active in the model and the imposed restoring meridional temperature gradient in the stratosphere is null (experiment C2), the model solution's stationary statistics exhibit a main jet near $45^{\circ} \mathrm{S}$, whose intensity increases with height all the way to the model's upper boundary (Fig. 3e). A weak secondary jet is noticeable around $25^{\circ} \mathrm{S}$ and easterlies characterize the equatorial troposphere.

Imposing a negative meridional temperature gradient on the upper model layers increases dramatically the similarity with the observations, as seen by comparing Fig. 3h with Figs. 1a,b: the main jet is now located near $50^{\circ} \mathrm{S}$ and its maximum values are at the tropopause, there is a weak secondary jet at about $25^{\circ} \mathrm{S}$, and easterlies dominate the equatorial regions up to the stratosphere. Model solutions for both the $\mathrm{C} 2$ and $\mathrm{C} 3$ experiments are characterized by synopticscale fluctuations (not shown here) that lead to an intermittent amplification of the secondary jet, especially in C3. Furthermore, close scrutiny of several years of the $\mathrm{C} 3$ experiment for $\Delta T=60 \mathrm{~K}$ indicates that the zonal wind anomalies at the tropopause level (not shown) propagate poleward with characteristic time scales very close to those found in $\mathrm{SH}$ observations (Koo et al. 2002).

It thus appears that in an otherwise greatly simplified atmospheric GCM subjected to radiative forcing and a temperature gradient reversal in the stratosphere, eddy dynamics help account for the observed tropospheric double-jet pattern, as well as for some of the intraseasonal variability described by Koo et al. (2002). Note that the number, position, and intensity of tropospheric jets strongly depends on the choice of parameters, in particular on the Rayleigh friction at the surface (Robinson 1997; Koo and Ghil 2002; Kravtsov et al. 2005; Bordi et al. 2007; Chen et al. 2007).

\section{c. Sensitivity study}

We study now the sensitivity of the primary and secondary circulation, as measured by the zonal-mean zonal wind pattern and the maximum intensity of the zonally averaged, meridional-plane streamfunction. To do so, the equator-to-pole restoring temperature difference $\Delta T_{r}(\sigma)$ in the troposphere is changed in steps of $10 \mathrm{~K}$, so that $\Delta T$ at the lowermost level varies from 40 to $80 \mathrm{~K}$; this range corresponds roughly to the observed seasonal range of the meridional temperature gradient at the surface.

The resulting zonal-mean zonal winds for $\Delta T=40 \mathrm{~K}$ and $\Delta T=70 \mathrm{~K}$ appear in Figs. 3a,d,g and Figs. 3c,f,i, respectively. In experiments $\mathrm{C} 1$ and $\mathrm{C} 2$, the changes are 

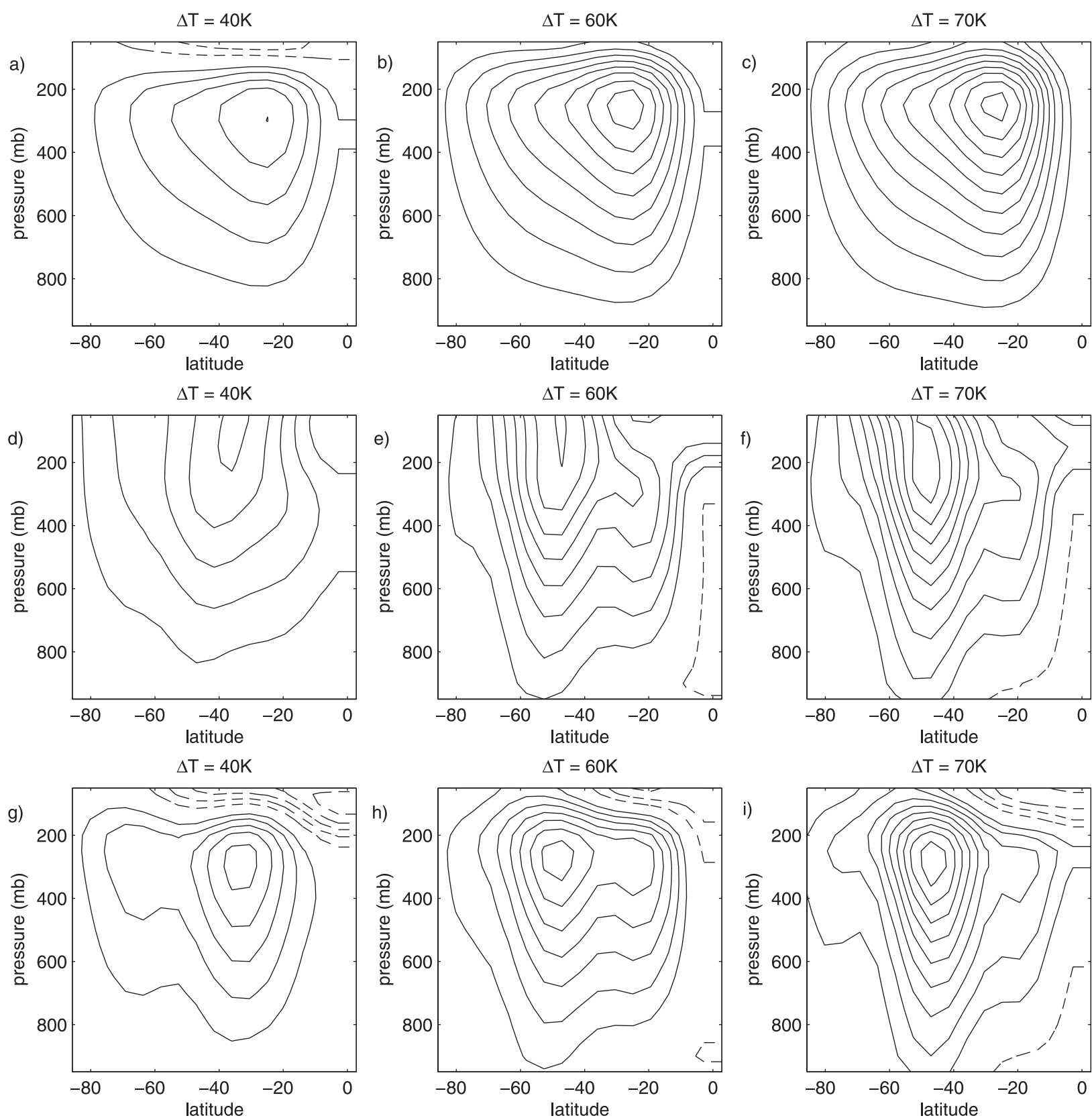

FIG. 3. PUMA simulations showing latitude-height (pressure) cross section of the zonal mean zonal wind for different values of temperature gradient at the lowermost level $\Delta T$, for experiments (a)-(c) $\mathrm{C} 1$, (d)-(f) $\mathrm{C} 2$, and (g)-(i) C3. Values of $\Delta T$ in all three experiments are taken successively equal to (a),(d),(g) 40, (b),(e),(h) 60, and (c),(f),(i) $70 \mathrm{~K}$; contours are as in Fig. 1.

mainly quantitative, whereas in $\mathrm{C} 3$ a change in regime is observed: the maximum upper-level westerly wind shifts from the subtropical to a midlatitude position as $\Delta T$ increases from $\Delta T=40 \mathrm{~K}$ to $\Delta T=60 \mathrm{~K}$ and beyond, whereas the reverse holds for the secondary maximum. This $\Delta T$ variation accompanies a dramatic change in the mean meridional circulation, as illustrated in Fig. 4.
The maximum intensities of the Hadley, Ferrell, and polar cells are plotted as a function of $\Delta T$ in Figs. $4 \mathrm{a}-\mathrm{c}$; in each one of the three experiments, the nature of the dependence on $\Delta T$ for the three cells is distinct. The mass streamfunctions of the three experiments are displayed in Figs. $4 \mathrm{~d}-\mathrm{f}$ for $\Delta T=40 \mathrm{~K}$.

In experiment $\mathrm{C} 1$, and only in $\mathrm{C} 1$, the dependence on $\Delta T$ of the maximum strength of the mass streamfunction is 

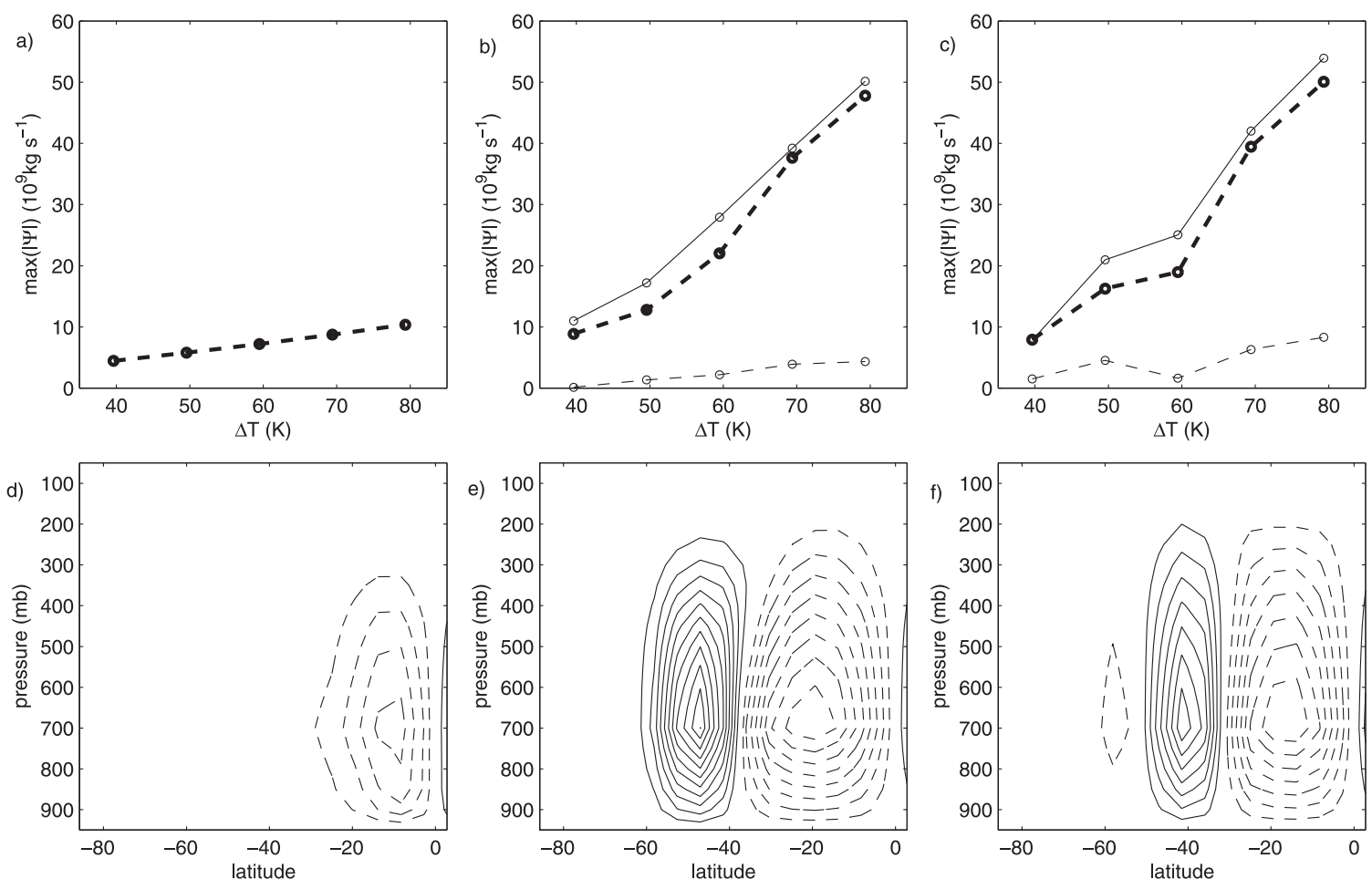

FIG. 4. PUMA sensitivity of the intensity of the mean meridional circulation on the changing equator-to-pole restoring temperature difference $\Delta T$. Maximum absolute values of the Hadley (heavy dashed line), Ferrell (light solid line), and polar (light dashed line) cells as a function of $\Delta T$ for the three cases: experiments (a) C1, (b) C2, and (c) C3. (d)-(f) Latitude-height (pressure) cross section of the mass streamfunction for $\Delta T=40 \mathrm{~K}$ for the three experiments, respectively; units are $10^{9} \mathrm{~kg} \mathrm{~s}^{-1}$ and contours are every $1 \times 10^{9} \mathrm{~kg} \mathrm{~s}^{-1}$, with solid lines denoting clockwise circulation.

almost linear and thus agrees with the results of Held and Hou (1980), in spite of our imposing a reverse temperature gradient in the stratosphere. Three remarkable differences among the experiments are noticeable from Fig. 4:

1) The Hadley cell in experiment $\mathrm{C} 1$ is quite weak compared to the others.

2) In the $C 3$ experiment, the slope for small $\Delta T$ (between 50 and $60 \mathrm{~K}$ ) is considerably less than for larger $\Delta T$; this difference in slope is less pronounced but still present in the $\mathrm{C} 2$ experiment. Such a difference suggests that the eddies, along with the meridional temperature gradient reversal in the stratosphere, are responsible for the nonlinear behavior of the maximum intensities of the three cells as a function of $\Delta T$.

3) Cases $\mathrm{C} 2$ and $\mathrm{C} 3$ show the emergence of the Ferrell and polar cells in accordance with observations.

The spatial patterns of the zonal-mean zonal wind in the $\mathrm{C} 3$ experiment do not change when vertical diffusion is included (not shown); this gives further support to the idea that the axisymmetric solution $(\mathrm{C} 1)$, although a possible solution of the PUMA model, is unstable with respect to the eddies.
In Fig. 5a we show the sensitivity of the conversion terms in the global mean Lorenz (1967) energy cycle of the PUMA model as a function of the equator-to-pole restoring temperature difference $\Delta T$ for experiments $\mathrm{C} 2$ and C3. The figure confirms the results described in Figs. $4 \mathrm{a}-\mathrm{c}$ : for the $\mathrm{C} 3$ experiment, there is an increase in the slope when $\Delta T$ is augmented from the range of $50-60 \mathrm{~K}$ to higher values; such a change is still present in the $\mathrm{C} 2$ experiment, although it is less pronounced in this case.

For all $\Delta T$ values and both experiments, the conversion terms that dominate are those from zonal potential to transient eddy potential energy, and then from eddy potential to kinetic energy of the transient eddies. This suggests that the double-jet structure and the regime change in the $\mathrm{C} 3$ experiment are related to baroclinic instability; in particular, a crucial role is played by synoptic waves with wavenumbers 4 to 9 . In addition, for experiment $\mathrm{C} 3(\Delta T=60 \mathrm{~K})$ we show in Figs. $5 \mathrm{~b}, \mathrm{c}$ the zonal-mean zonal wind for a 30-day interval. It is clear from Fig. $5 \mathrm{c}$ that the variability of the jets' maximum intensity occurs on a time scale longer than a month. This is probably due to the parameter setting used here, in particular to the length of the relaxation time for the 
a)

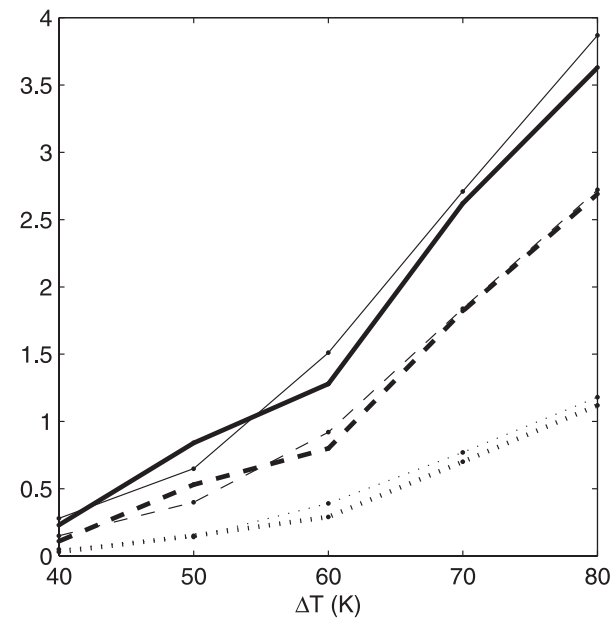

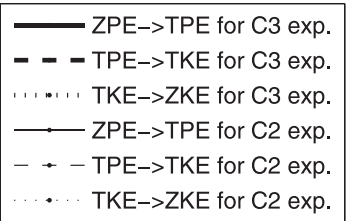

b)

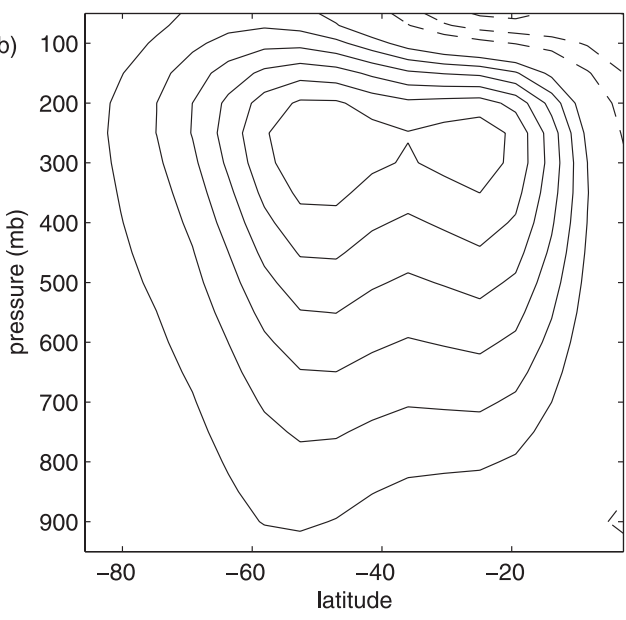

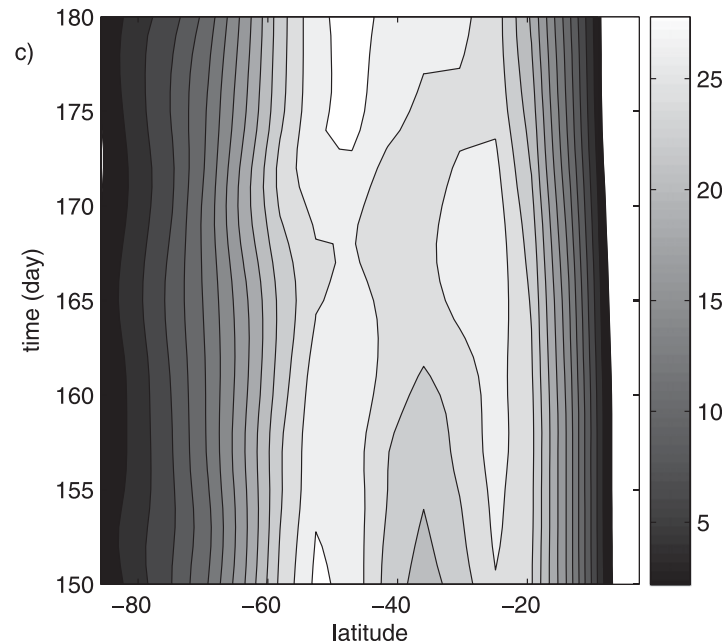

c)

FIG. 5. (a) PUMA sensitivity of the baroclinic life cycle conversion terms in the Lorenz energy cycle, as a function of $\Delta T$. Results are shown for experiments C2 (light lines) and C3 (bold lines): from zonal to transient eddy available potential energy (ZPE $\rightarrow$ TPE; solid), transient eddy available to transient eddy kinetic (TPE $\rightarrow$ TKE; dashed), and transient eddy to zonal kinetic energy (TKE $\rightarrow \mathrm{ZKE}$; dotted); units are $\mathrm{W} \mathrm{m}^{-2}$. (b) Latitude-height (pressure) cross section of the zonal wind averaged over days 150-180 for experiment C3 ( $\Delta T=60 \mathrm{~K})$. (c) Latitude-time diagram of the zonal mean zonal wind at $250 \mathrm{hPa}$. Units and contours are as in Fig. 2.
Newtonian forcing. These interesting observations will be further studied in future work.

The eddy heat fluxes in the $\mathrm{C} 2$ and $\mathrm{C} 3$ experiments are nonzero on average and vary on synoptic time scales, suggesting that baroclinic instabilities are active in giving rise to the double-jet pattern. Moreover, the zonal wind and eddy meridional heat fluxes at the latitude of the secondary jet ( $\mathrm{C} 3$ experiment) exhibit the vacillation mechanism typical of baroclinic instability when the wave-mean flow interaction is considered, as discussed by Bordi et al. (2006, hereafter BF06); see their Fig. 9 (not shown here).

Our model results further indicate that for weak imposed meridional temperature gradients in the troposphere, the circulation may undergo a spontaneous regime shift, depending on the sign of the temperature gradient in the stratosphere. Changes in model behavior when $\Delta T$ is varied suggest that the atmospheric circulation is quite sensitive to changes in radiative forcing. In particular, it seems that the instabilities and nonlinearities captured by the PUMA model inhibit the quick linear growth of the maximum intensity of the mass streamfunction when $\Delta T$ is varied in and near the interval 50-60 K.

The main factors that seem to be involved in the dynamics of the double-jet pattern are

(i) the baroclinic instability process with its heat and momentum fluxes induced by the eddies;

(ii) the radiative forcing in the stratosphere, with the associated reversal of the meridional temperature gradient; and 
(iii) nonlinear wave-mean flow and wave-wave interactions.

Recent work by Schneider and Walker (2006) suggests, however, that the interaction of the baroclinic eddies with the mean flow inhibits nonlinear eddy-eddy interactions that would engender an inverse energy cascade and questions the evidence for such a cascade in the earth's atmosphere (Boer and Shepherd 1983; Nastrom and Gage 1985; Straus and Ditlevsen 1999, and references therein). Although we do not necessarily agree with all the implications of this argument, it does justify to some extent our considering only the wavemean flow interaction.

\section{Dynamical interpretation: A nonlinear Eady model}

In this section we investigate the role of the eddy heat fluxes in generating the double-jet structure. Baroclinic eddies transport, in general, both heat and momentum. We wish to isolate the role of heat transfer to see whether it suffices, by itself, to generate an anomaly on the zonal wind that resembles the one discussed so far. The simplest framework in which this goal can be achieved is quasigeostrophic theory on an $f$ plane for a baroclinically unstable wave; indeed, the Eady model formulation does not allow any momentum transport. Moreover, to simplify the theoretical setting even further, we use only a single wave in a closed channel but allow for a zonal mean correction induced by the eddy field. Finally, we add to the traditional Eady model a layer of higher static stability to simulate stratospheric flow. The model is summarized below and details are provided in the appendix.

\section{a. Model description}

Following BF06, we consider a two-layer Eady model with the interface between the layers representing the tropopause. The model includes Ekman dissipation at the bottom boundary and Newtonian cooling at the surface and the tropopause. We take into account only the nonlinear interaction between the perturbation, described by a single wave in the zonal direction, and the mean flow, and we thus neglect the self-interaction of the perturbation. The flow is confined to a midlatitude channel of width $L$ and depth $H$, with $f$-plane geometry. The basic state has a constant density and constant vertical wind shear $\Lambda$; these assumptions are consistent with the Boussinesq approximation and a uniform meridional temperature gradient.

The nondimensional governing equations for conservation of potential vorticity $q$ and energy are given in log-pressure coordinates by

$$
\begin{aligned}
& \frac{d}{d t} q=0 \quad \text { and } \\
& \frac{d}{d t} \theta+S w=\Im
\end{aligned}
$$

here $q=\nabla_{H}^{2} \phi+f+\partial(\theta / S) / \partial z, \theta=\partial \phi / \partial z$ is the potential temperature, $S=N^{2} H^{2} /\left(f^{2} L^{2}\right)$ is the Burger number, $f$ the Coriolis parameter, $N$ the Brunt-Väisälä frequency, $\nabla_{H}^{2}$ the horizontal Laplacian operator, $w$ the vertical velocity, $\phi$ the total streamfunction, and $\Im$ the diabatic heating rate. The total streamfunction

$$
\phi=\phi_{b}(y, z)+\varphi(x, y, z, t)+\Phi(y, z, t)
$$

describes the basic state $\phi_{b}$, the wave field $\varphi$, and the zonal mean flow correction $\Phi$.

The Eady (1949) formulation of the baroclinic instability problem posits that $q_{\varphi}=q_{\Phi}=0$ (i.e., the potential vorticity of $\varphi$ and $\Phi$ is null everywhere, except at the surface, $z=0$, and at the top of the model atmosphere, $z=H_{T}$ ); thus, instability, if any, results solely from the temperature gradient along the boundaries. In the original Eady model, $z=H_{T}$ stands for the tropopause, whereas here we include two layers: one for the troposphere, $n=1$, and the other for the stratosphere, $n=2$. The basic state in both layers satisfies geostrophic equilibrium:

$$
\begin{aligned}
& U^{(1)}=U_{0}+\Lambda z \text { for } z \leq H_{T} \text { and } \\
& U^{(2)}=U^{(1)}\left(H_{T}\right)+a_{0} \Lambda\left(z-H_{T}\right) \text { for } z>H_{T},
\end{aligned}
$$

where superscripts $n=1,2$ denote the troposphere and the stratosphere, respectively, $U_{0}$ is the zonal wind at the surface, and $a_{0}$ is a parameter that accounts for the intensity and sign of the vertical wind shear in the stratosphere. With the assumptions above and substituting the total streamfunction in Eq. (2), we can write the equations for the zonal mean correction and the perturbation field (see BF06 and the appendix here). These equations include Ekman pumping at the ground and a diabatic heating rate parameterized in terms of $\theta$ as Newtonian cooling, with different relaxation time scales at the ground and at the tropopause.

In view of the horizontal boundary condition for $\varphi^{(n)}$ [Eq. (A4) in the appendix], we expand the wave field's meridional and vertical structure as follows:

$$
\varphi^{(n)}(x, y, z, t)=\sum_{k, l} \varphi_{k, l}^{(n)}(z, t) e^{i k x} \sin (l y)+\text { c.c.; }
$$

here $k$ and $l$ are the zonal and meridional wavenumbers, c.c. stands for the complex conjugate, and $\varphi_{k, l}^{(n)}(z, t)$ satisfy the corresponding interior equations, namely 
Eqs. (10)-(11) in BF06. A single zonal mode for the wave field allows only for wave-mean flow interaction, and the zonal mean flow correction $\Phi$ of Eq. (3) is given by

$$
\begin{aligned}
& \partial_{t z} \Phi^{(1)}+2 i k l \varphi_{k, l}^{(1)} \partial_{z} \varphi_{k, l}^{(1) *} \sin (2 l y)+\delta_{E} \partial_{y y} \Phi^{(1)} \\
& +\frac{1}{\tau_{0}} \partial_{z} \Phi^{(1)}=0 \quad \text { at } \quad z=0 \quad \text { and } \\
& \partial_{t z} \Phi^{(1)}+2 i k l \varphi_{k, l}^{(1)} \partial_{z} \varphi_{k, l}^{(1) *} \sin (2 l y)+\frac{1}{\tau_{H}} \partial_{z} \Phi^{(1)} \\
& \quad=\gamma\left[\partial_{t z} \Phi^{(2)}+\frac{1}{\tau_{H}} \partial_{z} \Phi^{(2)}\right] \text { at } z=H_{T}
\end{aligned}
$$

where the asterisk denotes the complex conjugate, $\gamma=$ $S^{(1)} / S^{(2)}$ the static stability ratio, $\delta_{E}=E^{1 / 2} S^{(1)} /\left(2 R_{0}\right)$ is the Ekman dissipation parameter (with $E$ and $R_{0}$ being the Ekman and Rossby numbers), and $\tau_{0}$ and $\tau_{H}$ are the restoration time scales at the surface and the tropopause, respectively. Notice that in the wide-channel limit $l \rightarrow 0$, Eq. (7) requires $\Phi^{(1)}=\Phi^{(2)}=0$. When $l$ is nonzero, the expansion $\sin (2 l y)=\left[2 /\left(l L_{y}\right)\right]$ $\sum_{j=1}^{\infty} g_{2 j-1} \cos [(2 j-1) l y]$, with $g_{2 j-1}=1 /\left[1-(j-0.5)^{2}\right]$, implies that jets should appear at the lateral boundaries.

We can, in fact, solve Eq. (7) by setting

$$
\Phi^{(n)}(y, z, t)=\sum_{l b} \Phi_{l b}^{(n)}(z, t) \cos (l b y),
$$

with $\Phi_{l b}^{(\mathrm{n})}(z, t)$ satisfying the interior equations. Unlike BF06, we include here the first two modes in the $y$ direction, $l_{b 1}=\pi / L_{y}$ and $l_{b 3}=3 \pi / L_{y}$, with $L_{y}$ being the meridional scale in the zonal mean correction.

\section{b. Sensitivity study}

By using the parameter settings described in the appendix and given the time behavior of the model coefficients in Eq. (A9), we obtain the zonal wind correction as

$$
\bar{U}^{(n)}(y, z, t)=-\frac{\partial}{\partial y} \Phi^{(n)}(y, z, t), \quad n=1,2 .
$$

The meridional structure consists of two terms: one is proportional to $\sin \left(\pi y / L_{y}\right)$ and is maximal at midchannel, while the other is proportional to $\sin \left(3 \pi y / L_{y}\right)$ and its maxima are not far from the lateral boundaries, leading to a double-jet pattern.

The meridional temperature gradient in the troposphere is controlled by the parameter $\Lambda$, whereas its reversal in the stratosphere is provided by the negative sign of $a_{0}$. The results for experiment C3 with PUMA in section 3 thus suggest computing solutions of our twolayer Eady model for different values of $\Lambda$ while keep- ing $a_{0} \Lambda$ constant in the stratosphere. Our reference values are $\Lambda=3$ and $a_{0}=-1.5\left(a_{0} \Lambda=-4.5\right)$, and we let $\Lambda$ vary from 1.2 to 10 with $a_{0}$ being changed accordingly; note that for $\Lambda<1.2$ the mean flow correction is zero.

Results for the zonal wind correction averaged over 2000 time units (i.e., 2000 days in dimensional time) for several $\Lambda$ values are shown in Figs. 6a-d. All the solutions in the figure exhibit a strong negative correction centered at midchannel, with maximum values at the tropopause. The midchannel maximum is due to the dominant contribution of $\sin \left(l_{b 1} y\right)$ to the zonal mean correction; this means that the basic state is strongly decelerated there. As the vertical wind shear increases, along with the meridional temperature gradient, from Figs. $6 \mathrm{a}$ to $6 \mathrm{~d}$, positive corrections occur near the lateral boundaries at the tropopause level and gradually accelerate the basic zonal wind there. This acceleration is related to the contribution of the second term $\sin \left(l_{b 3} y\right)$ in the zonal mean correction, which increases as $\Lambda$ increases.

A regime transition occurs between $\Lambda=1.8$ (not shown) and $\Lambda=2$ (Fig. 6c) and is related to the occurrence of the positive zonal mean correction near the lateral boundaries at the tropopause level. It is also confirmed by the projection of the solution trajectory onto the phase planes in Fig. 7; see Eq. (A9) for the exact definition of the pairs of expansion coefficients $\left(A_{0, l b 1}, B_{0, l b 1}\right)$ and $\left(A_{0, l b 3}, B_{0, l b 3}\right)$. For small values of the vertical wind shear, the two modes of the zonal mean correction have a single main frequency, whereas two main frequencies become detectable across the previously mentioned transition. Note that the trajectories occupy a larger portion of the phase space as $\Lambda$ increases, typical of a period-doubling transition (Jin and Ghil 1990; Koo and Ghil 2002; BF06). On the other hand, for $a_{0} \Lambda=-4.5$ in the stratosphere, the time means of the expansion coefficients $\left(A_{0, l b 1}, B_{0, l b 1}\right)$ and $\left(A_{0, l b 3}, B_{0, l b 3}\right)$ as a function of $\Lambda$ (Fig. 8 , light lines) have a pronounced change in slope between $\Lambda=1.8$ and $\Lambda=2$ that resembles the regime transition noticed in PUMA solutions (see Fig. 4c for experiment C3, with $\Delta T<0$ in the stratosphere). No such break in slope is apparent when considering $a_{0} \Lambda=0$ (heavy lines in Fig. 8), a fact that highlights the model's sensitivity to the meridional temperature gradient in the stratosphere.

To better understand the dynamics of the different solutions obtained, we display in Fig. 9 the total zonal wind by adding the basic state to the zonal mean correction of Fig. 6 . When the vertical wind shear in the troposphere $(\Lambda)$ is weak because $a_{0} \Lambda$ is negative and large, Eq. (5) leads to strong easterlies that dominate the stratosphere. The double-jet structure characterizes 

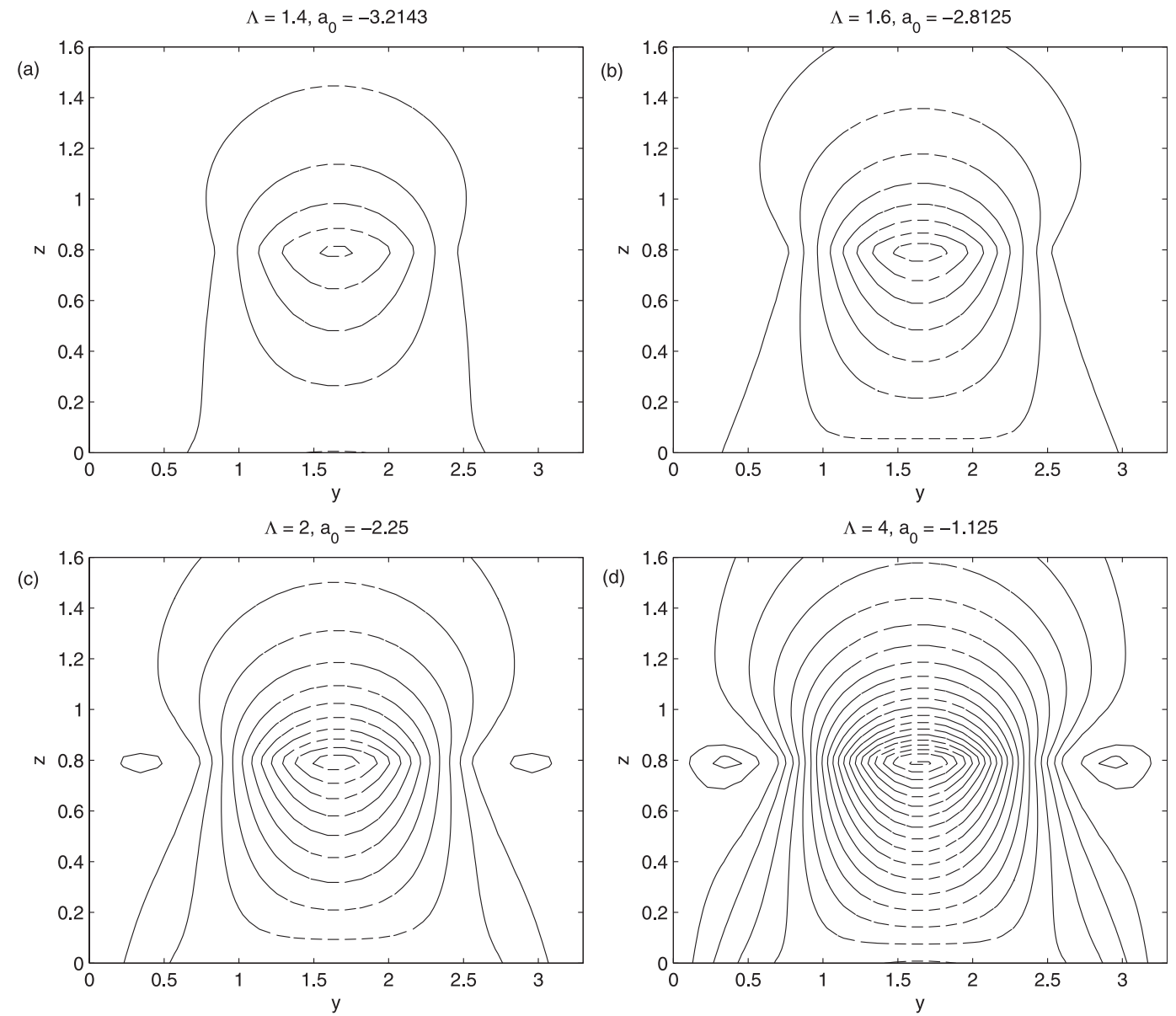

FIG. 6. Model solutions for the nonlinear, two-layer Eady model: $y-z$ cross section of the zonal wind correction $\bar{U}^{(n)}(y, z, t)$, where $n=1,2$, averaged over 2000 time units (2000 days in dimensional units), with vertical wind shear $\Lambda=$ (a) 1.4, (b) 1.6, (c) 2, and (d) 4, respectively, in the troposphere $(n=1)$, while $a_{0} \Lambda$ is kept constant in the stratosphere $(n=2)$. Values for $\Lambda$ and $a_{0}$ are indicated at the top of the each panel; the other parameters are as described in section 4. Units are dimensionless and contours are every 0.1 nondimensional unit; dashed lines denote negative values and the zero line is excluded.

the troposphere at all $\Lambda$ values shown, but the two jets are greatly intensified across the regime transition that occurs between $\Lambda=1.8$ and $\Lambda=2$. Note that for $a_{0}=0$, while all other parameters are unchanged (not shown), the coefficients of the zonal mean correction exhibit a single period in time for the entire range of $\Lambda$ values in Figs. 6 and 7; in this case, the corrections to the zonal flow are less intense, while the dependence of their spatial features on the vertical wind shear resembles the one seen in Fig. 6. Thus, with respect to the case $a_{0}=0$, the effects of introducing a reversed meridional temperature gradient in the stratosphere are twofold: amplifying the zonal mean corrections and introducing a different temporal variability, characterized by the presence of a regime change.

Thus, the results from our simple model show that baroclinic dynamics alone, by merely taking into ac- count wave-mean flow interaction in the presence of a temperature gradient reversal in the stratosphere, can give rise to a double-jet pattern in the troposphere. In particular, this confirms that eddy heat fluxes play a key role in establishing such a feature of the general circulation, as envisaged by Charney (1973).

\section{Concluding remarks}

\section{a. Summary}

Southern Hemisphere observations for the characteristic seasonal transition months of November and April show that the climatological zonal mean zonal wind is characterized by two maxima in the troposphere, whereas easterlies dominate the tropical-to-midlatitude stratosphere. Moreover, analysis of daily data suggests 
$\Lambda=1.4, a_{0}=-3.2143$

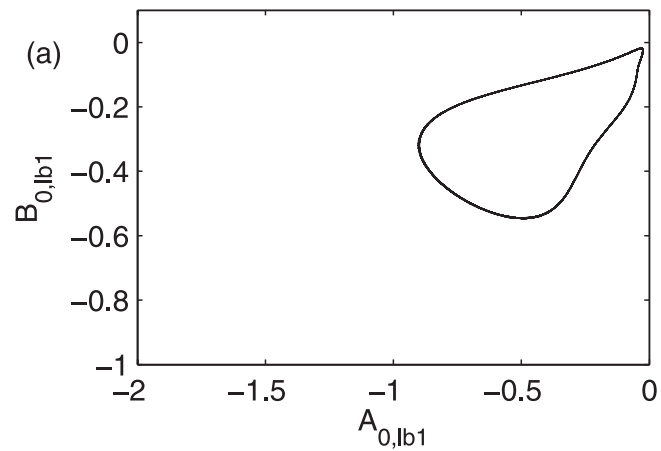

$\Lambda=1.6, a_{0}=-2.8125$

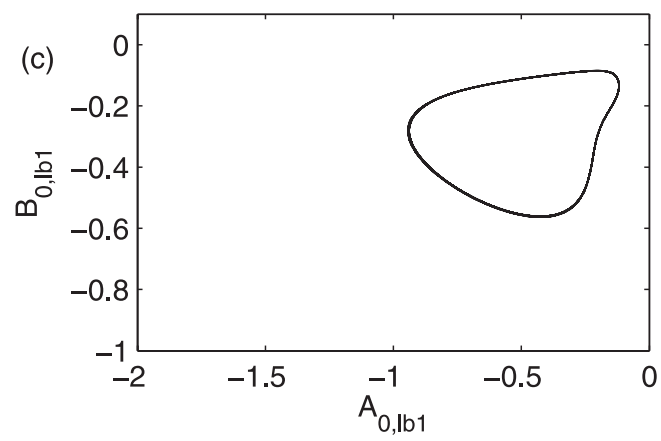

$\Lambda=2, a_{0}=-2.25$

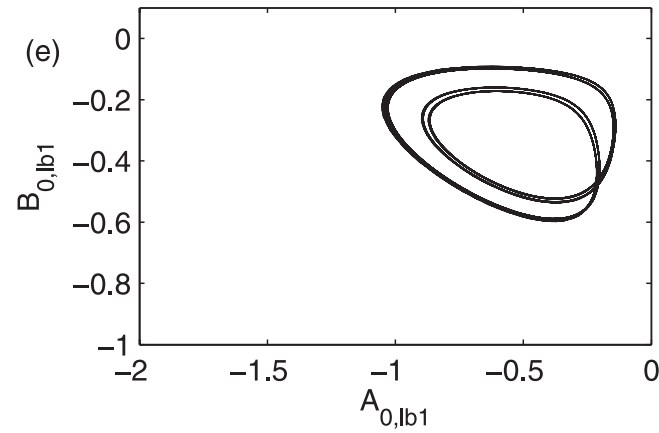

$\Lambda=4, a_{0}=-1.125$

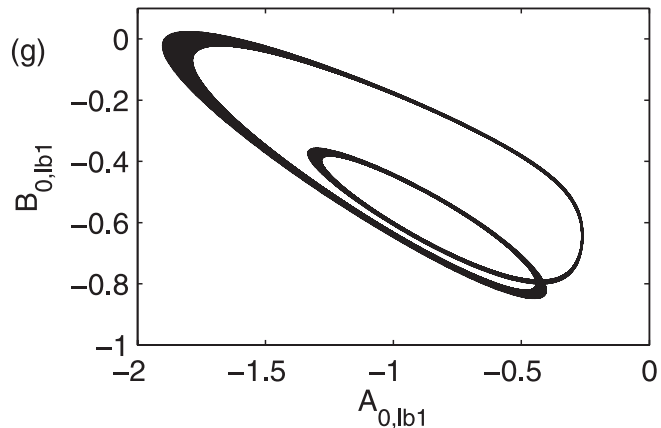

$\Lambda=1.4, a_{0}=-3.2143$
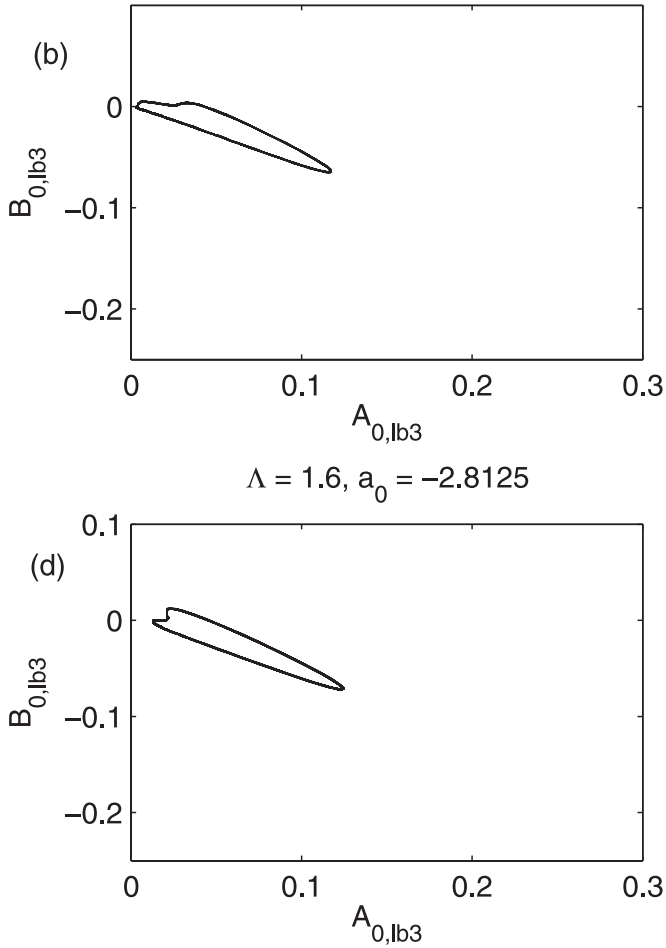

$\Lambda=2, a_{0}=-2.25$

(f)

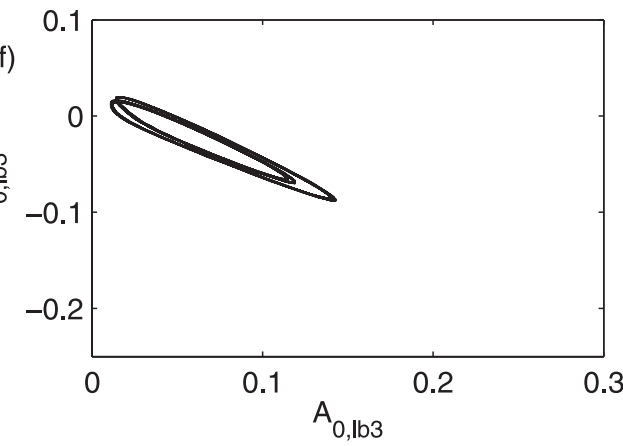

$\Lambda=4, a_{0}=-1.125$

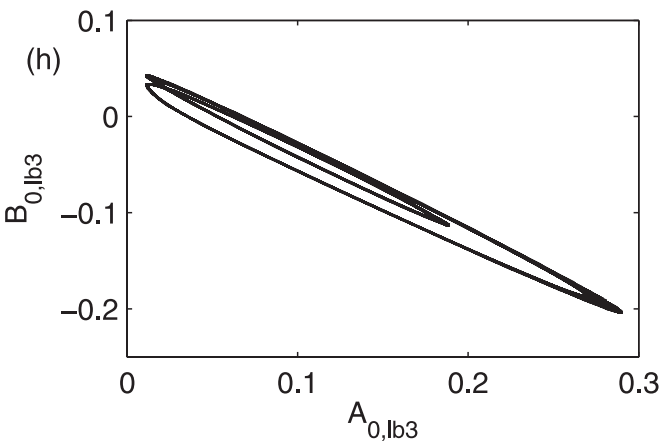

FIG. 7. Nonlinear Eady model solutions: projection of the solution trajectory onto the phase planes (a), (c), (e), (g) $\left(A_{0, l b 1}, B_{0, l b 1}\right)$ and (b), (d), (f), (h) $\left(A_{0, l b 3}, B_{0, l b 3}\right)$ for parameter settings illustrated in Fig. 6. Units are dimensionless. 
(a)

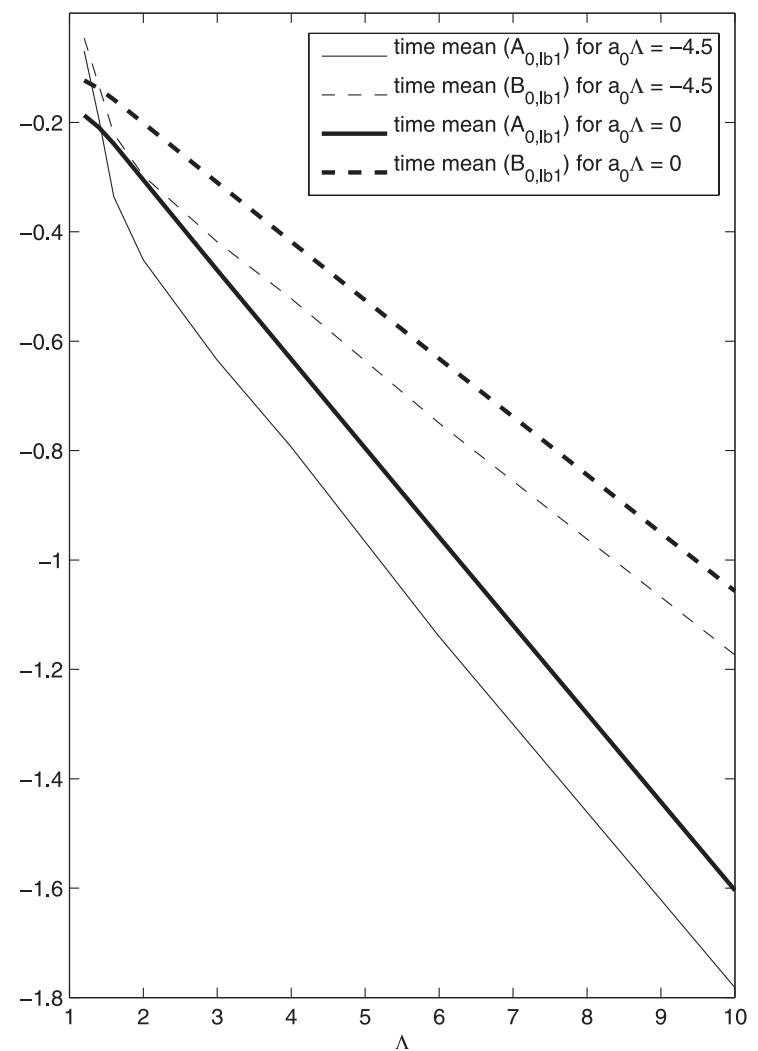

(b)

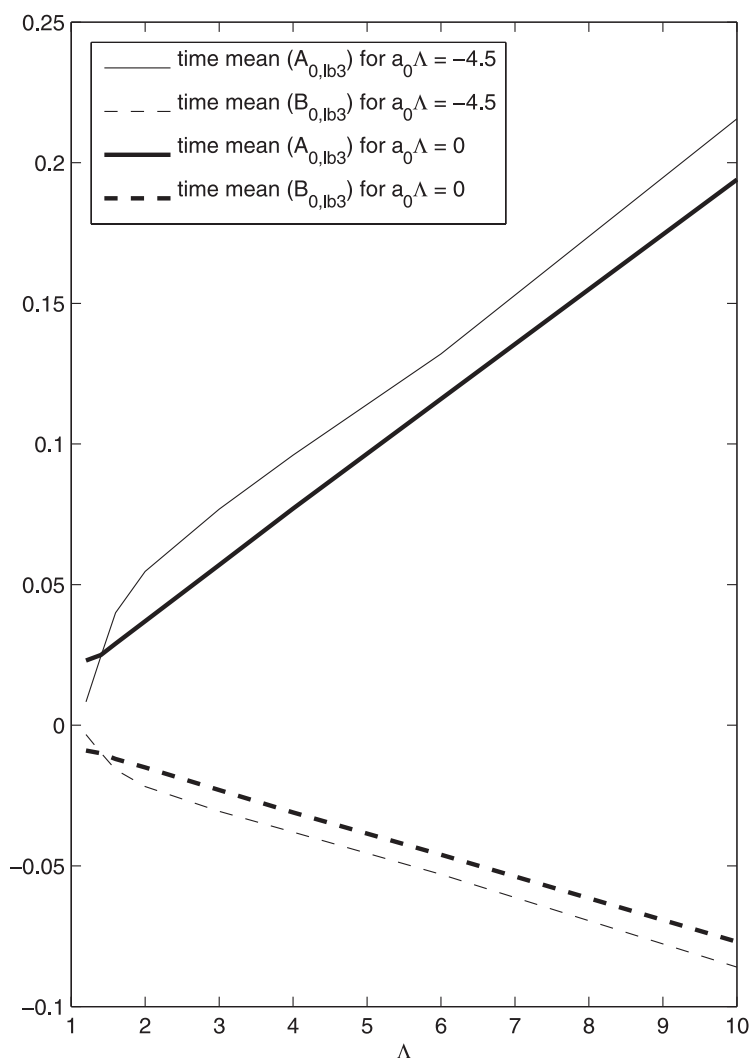

FIG. 8. Time mean of the expansion coefficients of Eq. (A9) as a function of the vertical wind shear $\Lambda$ in the troposphere, for $a_{0} \Lambda=-4.5$ (as in Fig. 6; light lines) and $a_{0} \Lambda=0$ (heavy lines) in the stratosphere: (a) $A_{0, l b 1}, B_{0, l b 1}$ and (b) $A_{0, l b 3}, B_{0, l b 3}$. Units are dimensionless; solid and dashed lines are explained in the legend.

that these prominent features of the general circulation are closely related to physical mechanisms that operate on synoptic time scales. Thus, the aim of the present study was to examine the effects of the eddies and the role of the stratosphere in producing a reasonable circulation in agreement with the observational evidence above.

For this purpose, we used both the simplified PUMA general circulation model and a nonlinear two-layer Eady model. PUMA experiments showed that for weak meridional temperature gradients in the troposphere, the model atmosphere may undergo a regime shift from a prevailing lower-latitude to a higher-latitude westerly jet, depending on the sign of the stratospheric temperature gradient. Furthermore, the model nonlinearities entail strong sensitivity of its circulation to changes in the radiative forcing.

Our weakly nonlinear two-layer Eady model, which only takes into account the nonlinear interaction between the perturbation and the mean flow, is able to capture the double-jet pattern. The two meridional modes considered for the zonal mean correction decelerate the basic zonal wind in the middle of the channel and accelerate it near the lateral channel boundaries, mainly at the tropopause level. Moreover-for a given parameter setting, and within the limitations of the model-it is possible to find, as a function of the vertical wind shear in the troposphere, a regime shift similar to that detected in PUMA simulations. Thus, it appears that the combined effects of the reversed meridional temperature gradient in the stratosphere and of the baroclinic eddies-acting through their heat transports and interactions with the mean flow-may explain, at least qualitatively, the observed prominent features of the $\mathrm{SH}$ general circulation during the transition seasons, namely the occurrence of easterlies in the stratosphere and of double jets in the troposphere.

Further analysis is required to illuminate the role played by the heat and momentum transports that are responsible for the observed behavior and so to verify the crucial effect of the heat fluxes, as suggested by model solutions. Furthermore, the behavior of the nonlinear 

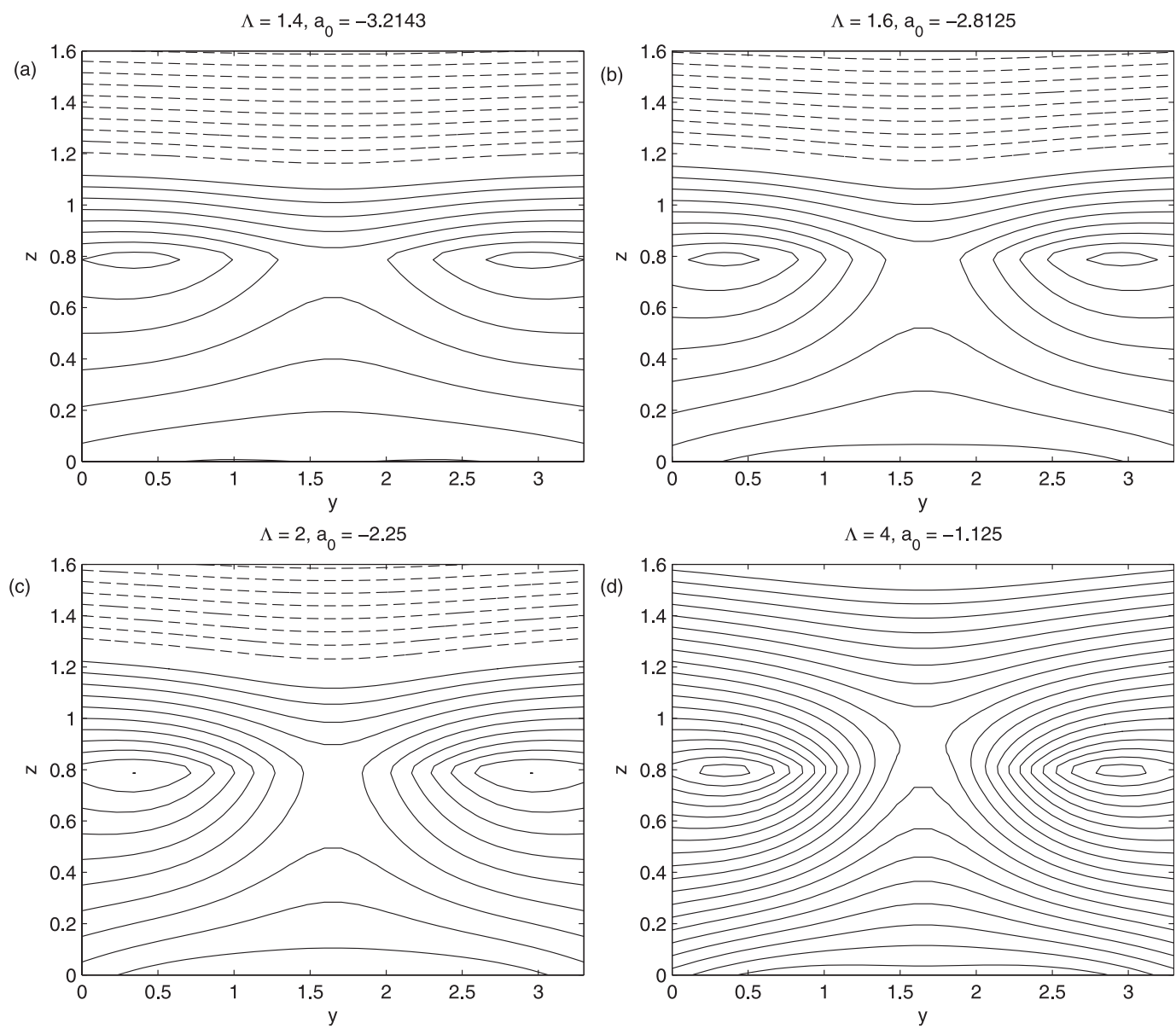

FIG. 9. Nonlinear Eady model solutions: $y-z$ cross section of the total zonal wind, basic state plus zonal mean correction for the parameter settings of Fig. 6. Contour interval is 0.2 nondimensional units; dashed lines denote negative values and the zero line is excluded.

Eady model should be studied systematically over a larger range of parameter values.

\section{b. Discussion}

A final comment may be in order concerning the main differences between our approach and that of the previous studies cited in the introduction. Here we study the origin of the double jet that in the $\mathrm{SH}$ is most noticeable on monthly mean maps of the two transition months of November and April. Previous studies, instead, were mainly focused on the low-frequency, intraseasonal variability of the zonal wind anomalies. We did find, however, that considering an extended multiyear run of the $\mathrm{C} 3$ experiment for $\Delta T=60 \mathrm{~K}$, zonal wind anomalies at the tropopause level do propagate poleward with characteristic time scales very close to those found in SH observations (Kidson 1988; Koo et al. 2002). This type of anomaly propagation is interrupted for weak $\Delta T$.
Furthermore, whereas we find that a regime change, from a prevailing subtropical jet to a midlatitude one, may occur as a function of the forcing temperature gradient in the troposphere (Figs. $3 \mathrm{~g}-\mathrm{i}$ ), previous studies found the two regimes, characterized by a high-latitude or a low-latitude jet, to exist for a fixed thermal forcing. The shifts between these two regimes are associated with the abovementioned propagation of zonal wind anomalies.

The two approaches thus complement each other because different features of the atmospheric circulation have been investigated: one (Koo and Ghil 2002; Kravtsov et al. 2005) studies the internal variability of the flow, with characteristic time scales of tens of days, and the other investigates changes induced by variations in tropospheric radiative forcing.

Acknowledgments. The ERA-40 data used in this study were obtained from the ECMWF data server (http:// www.ecmwf.int). It is a pleasure to thank F. Sielmann 
and F. Lunkeit (Hamburg University) for providing the Lorenz energy cycle analysis of the PUMA experiments, and two anonymous referees for numerous constructive suggestions. We gratefully acknowledge financial support provided by the European Commission's Project 12975 (NEST) "Extreme events: Causes and consequences (E2-C2)" (IB, MG, and AS), by the Office of Science (BER) of the U.S. Department of Energy, via Grant DE-FG02-07ER64439 (MG), by Project SFB-512 of the Deutsche Forschungsgemeinschaft (KF), and by a Max-Planck Fellowship.

\section{APPENDIX}

\section{Detailed Two-Layer Model Description}

We describe here in somewhat greater detail the nonlinear, two-layer Eady model with Ekman dissipation at the bottom boundary and a Newtonian cooling at the surface and the tropopause used in section 4 . This model differs from that of BF06 by the fact that two meridional modes are used in the zonal mean correction. This meridional structure allows the present model version to better reproduce the double-jet structure in the observations (section 2) and the simplified GCM simulations (section 3). We only allow a single zonal mode in the wave field and thus do not account for wave-wave interactions.

\section{a. Basic equations}

The governing equations in dimensionless form and $f$-plane geometry appear as Eq. (2) in the main text, along with the definition of all the symbols. They are repeated here for convenience:

$$
\begin{aligned}
& \frac{d}{d t} q=0 \quad \text { and } \\
& \frac{d}{d t} \theta+S w=\Im
\end{aligned}
$$

The total streamfunction is given by

$$
\phi=\phi_{b}(y, z)+\varphi(x, y, z, t)+\Phi(y, z, t),
$$

with $\phi_{b}$ being the basic state, $\varphi$ the wave field, and $\Phi$ the zonal mean flow correction.

\section{b. Two-layer formulation}

Two layers separated by the tropopause require the vertical velocity and streamfunction to be continuous at $z=H_{T}$, while the wave field and the mean flow correction vanish in the limit of $z$ going toward the outer space, $z \rightarrow \infty$. Each layer is characterized by constant static stability $S$. Denoting the troposphere and stratosphere by superscripts $n=1,2$, respectively, gives

$$
\begin{aligned}
& \bar{w}^{(1)}=0, \quad \tilde{w}^{(1)}=0, \quad \text { at } \quad z=0, \\
& \bar{w}^{(1)}=\bar{w}^{(2)}, \tilde{w}^{(1)}=\tilde{w}^{(2)}, \quad \text { at } \quad z=H_{T}, \\
& \Phi^{(1)}=\Phi^{(2)}, \varphi^{(1)}=\varphi^{(2)}, \quad \text { at } \quad z=H_{T}, \\
& \Phi^{(2)} \rightarrow 0, \quad \varphi^{(2)} \rightarrow 0, \text { for } \quad z \rightarrow \infty, \text { and } \\
& S=S^{(1)} \text { for } z<H_{T}, S=S^{(2)} \text { for } \quad z>H_{T} ;
\end{aligned}
$$

$\bar{w}^{(n)}$ and $\tilde{w}^{(n)}$ are the vertical velocities associated with the correction of the mean flow and the wave field, respectively.

\section{c. Boundary conditions}

The horizontal domain is zonally periodic and hence the meridional velocity must vanish at the lateral walls, whereas for the mean flow correction field $\Phi$, the zonal momentum equation requires that no zonally integrated acceleration can occur at the rigid $y$ boundaries; that is,

$$
\begin{gathered}
\partial_{x} \varphi^{(1)}=\partial_{x} \varphi^{(2)}=0 \quad \text { at } \quad y=0, L_{y} \quad \text { and } \\
\partial_{y t} \Phi^{(1)}=\partial_{y t} \Phi^{(2)}=0 \quad \text { at } \quad y=0, L_{y} .
\end{gathered}
$$

\section{d. Basic state}

The basic state, which satisfies the geostrophic constraint, is given by

$$
\begin{aligned}
& U^{(1)}=U_{0}+\Lambda z \text { for } z \leq H_{T} \text { and } \\
& U^{(2)}=U^{(1)}\left(H_{T}\right)+a_{0} \Lambda\left(z-H_{T}\right) \text { for } z>H_{T},
\end{aligned}
$$

where $U_{0}$ is the zonal wind at the surface and $a_{0}$ a parameter that accounts for the intensity and sign of the vertical wind shear in the stratosphere. With the assumptions above and substituting the total streamfunction in Eq. (A1), we can write the equations for the zonal mean correction and the perturbation field; see Eqs. (5) and (6), (8)-(11), and (13) and (14) of BF06.

\section{$e$. The wave field and zonal mean correction}

Let us consider the meridional and vertical structure of the wave field and the zonal mean correction. In view of the horizontal boundary condition for $\varphi^{(n)}$ in Eq. (A4), we expand the wave field as follows:

$$
\varphi^{(n)}(x, y, z, t)=\sum_{k, l} \varphi_{k, l}^{(n)}(z, t) e^{i k x} \sin (l y)+\text { c.c. },
$$


where all quantities have been defined in the main text. As in BF06, we select a single zonal eddy mode (i.e., we consider only the wave-mean flow interaction). In this case, the equations for the zonal mean correction appear as Eq. (7) in section 4.
By using here the first two meridional modes $l_{b 1}=$ $\pi / L_{y}$ and $l_{b 3}=3 \pi / L_{y}$ of the expansion $\sin (2 l y)=$ $\left[2 /\left(l L_{y}\right)\right] \Sigma_{j=1}^{\infty} g_{2 j-1} \cos [(2 j-1) l y]$, with $g_{2 j-1}=1 /[1-$ $\left.(j-0.5)^{2}\right]$, we can solve Eq. (7) in the following form:

$$
\begin{gathered}
\Phi^{(1)}(y, z, t)=\left[A_{0, l_{b 1}}(t) \sinh \left(\alpha_{0, l_{b 1}}^{(1)} z\right)+B_{0, l_{b 1}}(t) \cosh \left(\alpha_{0, l_{b 1}}^{(1)} z\right)\right] \sqrt{\frac{2}{L_{y}}} \cos \left(l_{b 1} y\right) \\
+\left[A_{0, l_{b 3}}(t) \sinh \left(\alpha_{0, l_{b 1}}^{(2)} z\right)+B_{0, l_{b 3}}(t) \cosh \left(\alpha_{0, l_{b 1}}^{(2)} z\right)\right] \sqrt{\frac{2}{L_{y}}} \cos \left(l_{b 3} y\right), \\
\Phi^{(2)}(y, z, t)=D_{0, l_{b 1}}(t) e^{-\alpha_{0, l_{b 1}}^{(2)} z} \sqrt{\frac{2}{L_{y}}} \cos \left(l_{b 1} y\right)+D_{0, l_{b 3}}(t) e^{-\alpha_{0, l_{b 3}}^{(2)} z} \sqrt{\frac{2}{L_{y}}} \cos \left(l_{b 3} y\right) ; \\
\varphi^{(1)}(x, y, z, t)=\left[A_{k, l}(t) \sinh \left(\alpha_{k, l}^{(1)} z\right)+B_{k, l}(t) \cosh \left(\alpha_{k, l}^{(1)} z\right)\right] \sqrt{\frac{2}{L_{y}}} \sqrt{\frac{1}{L_{x}}} \sin (l y) e^{i k(x-c t)}+\text { c.c. and } \\
\varphi^{(2)}(x, y, z, t)=D_{k, l}(t) e^{-\alpha_{k, l}^{(2)} z} \sqrt{\frac{2}{L_{y}}} \sqrt{\frac{1}{L_{x}}} \sin (l y) e^{i k(x-c t)}+\text { c.c. }
\end{gathered}
$$

Here $c$ is the phase speed of the wave; $\alpha^{(n)}{ }_{k, l}=$ $\left[\left(k^{2}+l^{2}\right) S^{(n)}\right]^{1 / 2}, \alpha_{0, l b 1}^{(n)}=l_{b 1} S^{(n) 1 / 2}$, and $\alpha^{(n)}{ }_{0, l b 3}=$ $l_{b 3} S^{(n) 1 / 2}$; and $A_{k, l}$ and $B_{k, l}$ are complex functions of time, while $A_{0, l b 1}, A_{0, l b 3}, B_{0, l b 1}$, and $B_{0, l b 3}$ are real functions. By using the continuity condition of the streamfunction at the tropopause, the coefficients $D_{(n)}(t)$ can be eliminated from Eqs. (A9) and (A10).

By substituting expressions (A9) and (A10) into the thermodynamic equations for the zonal mean correction and the wave dynamics [Eqs. (8) and (9), and (13) and (14), respectively, of BF06] and projecting them onto the basis functions, we obtain the equations for the time evolution of the coefficients $A_{0, l b 1}, B_{0, l b 1}, A_{0, l b 3}$, $B_{0, l b 3}, A_{k, l}$, and $B_{k, l}$. Finally, we integrate the system by using a leapfrog scheme.

\section{f. Parameter settings}

We set $U=10 \mathrm{~m} \mathrm{~s}^{-1}, L=10^{6} \mathrm{~m}$, and $H=10^{4} \mathrm{~m}$ for the scaling of the dimensional variables. We take the usually accepted value of the stratification ratio $\gamma=1 / 4$ and fix the other free parameters to Earthlike values as follows: $k=2 \pi s / L_{x}, l=\pi m / L_{y}, L_{x}=2 \pi r_{a} \cos \left(\theta_{0}\right), L_{y}=$ $r_{a} \Delta \theta$ (with $r_{a}$ being the nondimensional Earth radius), $\theta_{0}=45^{\circ}, \Delta \theta=30^{\circ}, m=1, U_{0}=0.5, \delta_{E}=0.1, \tau_{0}=3$, and $\tau_{H}=10$ time units. We let the nondimensional tropopause height be $H_{T}=0.8$ and choose the nondimensional zonal wavenumber $s$ be close to the most unstable wave obtained from the linear Eady problem with a rigid lid at such a tropopause height, namely $s=5$. The parameters $\Lambda$ and $a_{0}$ are varied as described in section 4 .

For the meridional structure of the wave field, we select $m=1$ - that is, the most unstable mode, which is symmetric with respect to the middle of the channel and satisfies the boundary conditions (A4). For the antisymmetric mode $m=2$, it is easy to show that the contributions of the eddy heat fluxes in Eq. (7) differ from zero only for $l_{b}=m_{b} \pi / L_{y}$, with $m_{b}$ being odd. Thus, by projecting the model equations onto the basis functions $\cos \left(l_{b 1} y\right)$ and $\cos \left(l_{b 3} y\right)$, we obtain the same set of equations for the time evolution of the coefficients, apart from the constants in the heat-flux terms being different. In this case, for the same parameter settings and for the values of $\Lambda$ and $a_{0}$ under consideration, we always obtain steady-state solutions. Because the mode $m=2$ is less unstable, the associated heat fluxes are weaker and are thus not able to give rise to double jets, in agreement with the observations.

\section{g. Zonal mean correction}

Given the time evolution of the model coefficients $A_{0, l b 1}, B_{0, l b 1}, A_{0, l b 3}$, and $B_{0, l b 3}$, we can compute the zonal wind correction in the two layers as

$$
\bar{U}^{(n)}(y, z, t)=-\frac{\partial}{\partial y} \Phi^{(n)}(y, z, t) .
$$




\section{REFERENCES}

Akahori, K., and S. Yoden, 1997: Zonal flow vacillation and bimodality of baroclinic eddy life cycles in a simple global circulation model. J. Atmos. Sci., 54, 2349-2361.

Boer, G. J., and T. G. Shepherd, 1983: Large-scale two-dimensional turbulence in the atmosphere. J. Atmos. Sci., 40, 164-184.

Bordi, I., A. Dell'Aquila, A. Speranza, and A. Sutera, 2002: Formula for a baroclinic adjustment theory of climate. Tellus, 54A, 260-272.

_ , K. Fraedrich, F. Lunkeit, and A. Sutera, 2006: On non-linear baroclinic adjustment with the stratosphere. Nuovo Cimento, 29C, 497-518.

,,--- , and $\longrightarrow, 2007$ : Tropospheric double-jets, meridional cells and eddies: A case study and idealized simulations. Mon. Wea. Rev., 135, 3118-3133.

Charney, J. G., 1973: Planetary fluid dynamics. Dynamic Meteorology, P. Morel, Ed., Reidel, 99-351.

Chen, G., I. M. Held, and W. A. Robinson, 2007: Sensitivity of the latitude of the surface westerlies to surface friction. J. Atmos. Sci., 64, 2899-2915.

Dima, J. M., and J. M. Wallace, 2003: On the seasonality of the Hadley cell. J. Atmos. Sci., 60, 1522-1527.

Eady, E. T., 1949: Long waves and cyclone waves. Tellus, 1, 33-52.

Farrell, B. F., and P. J. Ioannou, 2003: Structural stability of turbulent jets. J. Atmos. Sci., 60, 2101-2118.

Fraedrich, K., E. Kirk, and F. Lunkeit, 1998: Portable University Model of the Atmosphere. Deutsches Klimarechenzentrum Tech. Rep. 16, 38 pp.

— - _ U. Luksch, and F. Lunkeit, 2005: The Portable University Model of the Atmosphere (PUMA): Storm track dynamics and low-frequency variability. Meteor. Z., 14, 735-745.

Gallego, D., P. Ribera, R. Garcia-Herrera, E. Hernandez, and L. Gimeno, 2005: A new look for the Southern Hemisphere jet stream. Climate Dyn., 24, 607-621.

Galperin, B., S. Sukoriansky, N. Dikovskaya, P. L. Read, Y. H. Yamazaki, and R. Wordsworth, 2006: Anisotropic turbulence and zonal jets in rotating flows with a $\beta$-effect. Nonlinear Processes Geophys., 13, 83-98.

Hartmann, D. L., and F. Lo, 1998: Wave-driven zonal flow vacillation in the Southern Hemisphere. J. Atmos. Sci., 55, 1303 1315.

Held, I. M., and A. Y. Hou, 1980: Nonlinear axially symmetric circulations in a nearly inviscid atmosphere. J. Atmos. Sci., 37, 515-533.

_ , and M. J. Suarez, 1994: A proposal for the intercomparison of the dynamical cores of atmospheric general circulation models. Bull. Amer. Meteor. Soc., 75, 1825-1830.

Hoskins, B. J., and A. J. Simmons, 1975: A multi-layer spectral model and the semi-implicit method. Quart. J. Roy. Meteor. Soc., 101, 637-655.

James, I. N., and L. J. Gray, 1986: Concerning the effect of surface drag on the circulation of a planetary atmosphere. Quart. J. Roy. Meteor. Soc., 112, 1231-1250.

Jin, F.-F., and M. Ghil, 1990: Intraseasonal oscillations in the extratropics: Hopf bifurcation and topographic instabilities. $J$. Atmos. Sci., 47, 3007-3022.

Kalnay, E., and Coauthors, 1996: The NCEP/NCAR 40-Year Reanalysis Project. Bull. Amer. Meteor. Soc., 77, 437-471.
Kidson, J. W., 1988: Indices of the Southern Hemisphere zonal wind. J. Climate, 1, 183-194.

Koo, S., and M. Ghil, 2002: Successive bifurcations in a simple model of atmospheric zonal-flow vacillation. Chaos, 12, 300309.

_ - A. W. Robertson, and M. Ghil, 2002: Multiple regimes and low-frequency oscillations in the Southern Hemisphere's zonal mean flow. J. Geophys. Res., 107, 4596, doi:10.1029/ 2001JD001353.

Kravtsov, S., A. W. Robertson, and M. Ghil, 2005: Bimodal behavior in the zonal mean flow of a baroclinic $\beta$-channel model. J. Atmos. Sci., 62, 1746-1769.

$\longrightarrow,-$, and _ 2006: Multiple regimes and low-frequency oscillations in the Northern Hemisphere's zonal mean flow. $J$. Atmos. Sci., 63, 840-860.

Kushner, P. J., I. M. Held, and T. L. Delworth, 2001: Southern Hemisphere atmospheric circulation response to global warming. J. Climate, 14, 2238-2249.

Lorenz, E. N., 1963: The mechanics of vacillation. J. Atmos. Sci., 20, 448-465.

- 1967: The Nature and Theory of the General Circulation of the Atmosphere. World Meteorological Organization, $161 \mathrm{pp}$.

Mo, K. C., and M. Ghil, 1987: Statistics and dynamics of persistent anomalies. J. Atmos. Sci., 44, 877-901.

Namias, J., 1950: The index cycle and its role in the general circulation. J. Meteor., 7, 130-139.

Nastrom, G. D., and K. S. Gage, 1985: A climatology of atmospheric wavenumber spectra of wind and temperature observed by commercial aircraft. J. Atmos. Sci., 42, 950-960.

Panetta, R. L., 1993: Zonal jets in wide baroclinically unstable regions: Persistence and scale selection. J. Atmos. Sci., 50, 2073-2106.

Robinson, W. A., 1997: Dissipation dependence of the jet latitude. J. Climate, 10, 176-182.

Rossby, C.-G., and Coauthors, 1939: Relation between variations in the intensity of the zonal circulation of the atmosphere and the displacements of the semi-permanent centers of action. $J$. Mar. Res., 2, 38-55.

Schneider, T., and C. C. Walker, 2006: Self-organization of atmospheric macroturbulence into critical states of weak nonlinear eddy-eddy interactions. J. Atmos. Sci., 63, 15691586

Stone, D. A., A. J. Weaver, and R. J. Stouffer, 2001: Projection of climate change onto modes of atmospheric variability. $J$. Climate, 14, 3551-3565.

Straus, D. M., and P. Ditlevsen, 1999: Two-dimensional turbulence properties of the ECMWF reanalyses. Tellus, $\mathbf{5 1 A}$, 749-772.

Uppala, S. M., and Coauthors, 2005: The ERA-40 Re-Analysis. Quart. J. Roy. Meteor. Soc., 131, 2961-3012.

Williams, G. P., 2003: Jovian dynamics. Part III: Multiple, migrating, and equatorial jets. J. Atmos. Sci., 60, 1270-1296.

Yoden, S., M. Shiotani, and I. Hirota, 1987: Multiple planetary flow regimes in the Southern Hemisphere. J. Meteor. Soc. Japan, 65, 571-585.

Yu, J.-Y., and D. L. Hartmann, 1993: Zonal flow vacillation and eddy forcing in a simple GCM of the atmosphere. J. Atmos. Sci., 50, 3244-3259. 